\title{
Um Estudo de Caso com o Programa Parental ACT para Educar Crianças em Ambientes Seguros
}

\author{
Jéssica de Assis Silva ${ }^{1}$ \\ Programa de Pós-Graduação em Psicologia da Universidade Federal de São Carlos, \\ São Carlos, SP, Brasil \\ Lúcia Cavalcanti de Albuquerque Williams \\ Departamento de Psicologia da Universidade Federal de São Carlos, São Carlos, SP, Brasil
}

\begin{abstract}
Resumo
O objetivo deste artigo é relatar um estudo de caso descrevendo uma intervenção feita a uma mãe com o Programa ACT para Educar Crianças em Ambientes Seguros - programa de prevenção universal à violência contra a criança Tal estudo avaliou características comportamentais de uma mãe e seu filho de seis anos, com medidas de pré-teste/pós-teste/follow-up. A mãe preencheu o Inventário de Estilos Parentais e os instrumentos ASEBA (CBCL, ASR e ABCL) antes e após a intervenção e no followup, além do Questionário de Avaliação do Programa ACT após o treino. O marido, informante eleito pela participante preencheu o CBCL e o ABCL em todas as fases do estudo. Os resultados apontaram para uma mudança no estilo parental do cuidador após intervenção (de "Regular Acima da Média" para "Ótimo"). Houve concordância entre os informantes quanto ao comportamento da criança e discordância quanto ao comportamento da participante. $\mathrm{O}$ fato de o casal ter decidido se separar ao longo da intervenção dificultou uma interpretação precisa de tais discordâncias. Intervenções em grupo envolvendo a aplicação do Programa ACT no Brasil são necessárias.
\end{abstract}

Palavras-chave: Violência contra a criança, prevenção universal, programa ACT.

\section{A Case Study with the ACT Raising Safe Kids Parenting Program}

\begin{abstract}
The aim of this paper is to describe a case study of an intervention conducted with a Brazilian mother using the ACT Raising Safe Kids Program - a universal program to prevent violence against children. This study evaluated behavioral aspects of a mother, and her six-year old son in pre-post intervention measures, and follow-up. The mother filled the Brazilian instrument Parental Style Inventory (IEP), as well as the ASEBA (CBCL, ASR and ABCL) prior and after the intervention, and at follow-up; in addition to the ACT Program Assessment Questionnaire after the intervention. The husband filled the CBCL and the ABCL in all the study's phases. Results pointed to a change in parental style after intervention (from "High Average" to "Excellent"). There was agreement between informants about their son's behavior, and disagreement between spouses about mother's behaviors. The fact that the couple
\end{abstract}

1 Endereço para correspondência: Universidade Federal de São Carlos, Departamento de Psicologia, Laboratório de Análise e Prevenção da Violência, Rodovia Washington Luís, km235, São Carlos, SP, Brasil 13565-905. E-mail: jehpsi87@gmail.com e williams@ufscar.br

$\mathrm{O}$ artigo obteve apoio da Fundação de Amparo à Pesquisa do Estado de São Paulo (FAPESP/ Processo 2013/10417-6) e o projeto em que se insere foi submetido ao Comitê de Ética da Universidade Federal de São Carlos (processo 277.394). 
decided to separate during the intervention adds challenges to an accurate interpretation of the couple's discrepant results. Studies involving group application of the ACT Program are needed in Brazil.

Keywords: Child abuse, universal prevention, ACT program.

\section{Un Estudio de Caso utilizando el Programa ACT de Prevención de Violencia para las Familias}

\section{Resumen}

Este trabajo presenta un caso de estudio que describe una intervención realizada a una madre con el Programa de ACT para Educar Niños en Ambientes Seguros - programa de prevención universal a la violencia contra los niños. El estudio evaluó las características de comportamiento de una madre y su niño de seis años con medidas antes y después de la intervención y seguimiento. La madre llenaba el Inventario de Estilos Parentales y los instrumentos ASEBA (CBCL, ASR, ABCL) - antes y después de la intervención y en el seguimiento, más el Cuestionario de Evaluación del Programa ACT después de la intervención. El marido de la participante llenó el CBCL y el ABCL en todas las fases del estudio. Los resultados apuntan a un cambio de estilo parental después de la intervención (de "Encima del Promedio Normal" a "Excelente"). Hubo acuerdo entre los informantes acerca de la conducta del niño y el desacuerdo acerca de la conducta de la participante. El hecho de que la pareja ha decidido separarse durante la intervención dificulta una interpretación precisa de los resultados discrepantes. Estudios con la aplicación en grupo del Programa de ACT, son necesarios en Brasil.

Palabras clave: Violencia contra los niños, prevención universal, programa ACT.

A violência contra a criança é um problema comum ao redor do mundo e seus efeitos podem persistir na idade adulta, com possíveis consequências físicas, sociais e de saúde mental às vítimas (Dubowitz, 2012). É consenso que crianças e adolescentes, especialmente aqueles que crescem em lares tidos como violentos, devem ser um foco importante em projetos de prevenção (Wolfe \& Jaffe, 1999). No entanto, ainda são poucos os programas desenvolvidos e bem avaliados focados em cuidados primários de crianças (Dubowitz, 2012).

Um treinamento comportamental realizado diretamente com as crianças sem que haja um trabalho com os cuidadores e sem articulação de fatores ambientais tem sucesso limitado (Knox, Burkhart, \& Howe, 2011). Knox et al. (2011) argumentam que é preferível que seja feita uma abordagem educativa aos pais antes que haja o enraizamento de problemas comportamentais não-adaptativos nas crianças resultante de práticas parentais ineficazes e coercitivas. Gomide (2003) ressalta que práticas educativas parentais podem favorecer o desenvolvimento de compor- tamentos antissociais (resultante de abuso físico, psicológico, negligência e punição inconstante, por exemplo) ou pró-sociais (resultantes de monitoria positiva e comportamento moral), a depender de sua frequência e qualidade. Assim, o tipo de relação parental estabelecida parece ser fundamental no processo de desenvolvimento da criança (Carvalho \& Gomide, 2005). Uma intervenção cujo foco seja direcionado à maneira de agir dos cuidadores passa a ser fundamental, uma vez que pode significar uma alternativa de melhora na qualidade de vida intrafamiliar, interferindo positivamente, inclusive, na diminuição de problemas individuais dos pais, como a depressão (Prada \& Williams, 2010).

Para Wolfe e Jaffe (1999), promover atitudes e comportamentos incompatíveis com a violência e o abuso e encorajar a formação de relações saudáveis e não violentas refletiria uma maneira de se aproximar aos objetivos da prevenção. É consenso na literatura (Rios \& Williams, 2008) de que práticas parentais positivas (sem o uso de violência física e psicológica) estariam relacionadas ao desenvolvimento saudável da crian- 
ça e a adoção de tais práticas na relação pais/ filhos seria passo fundamental na prevenção de padrões intergeracionais de violência familiar, auxiliando a promoção de competência e comportamento adaptativo da criança.

A Organização Mundial de Saúde (OMS) World Health Organization (2009), fez um apelo no sentido de prevenir dificuldades comportamentais e violência infantil por meio do desenvolvimento de relações saudáveis entre crianças e seus cuidadores. Nesse sentido, os programas parentais têm sido designados a fornecer suporte por meio do treinamento de habilidades parentais para garantir o bem-estar da criança (Mejia, Calam, \& Sanders, 2012). Tal treino corresponde a uma ferramenta importante de orientação a pais e cuidadores para disciplinar crianças e adolescentes, objetivando a promoção de comportamentos adequados em seus filhos e favorecendo sua socialização (Bolsoni-Silva \& Marturano, 2002; Carvalho \& Gomide, 2005).

Os programas parentais têm se mostrado estratégias preventivas eficazes em países desenvolvidos, entretanto, os resultados de estudos relativos à sua eficácia em países em desenvolvimento ainda são limitados, havendo inclusive obstáculos na determinação de prevalência de dificuldades comportamentais e emocionais nesses países (Mejia et al., 2012). Em estudo de revisão internacional Prada e Williams (2010) relembram que durante a década de 1960, os programas de intervenção parentais seguiam o modelo triádico, no qual o terapeuta seria um mediador entre os pais e a criança na redução dos problemas comportamentais. Durante a década de $70, \mathrm{o}$ foco dos programas parentais desenvolvidos seria em problemas comportamentais específicos, como os de comportamento opositor da criança. Lançando o olhar sob a década de 80 , estudos realizados passaram a levar em consideração características parentais enquanto variáveis influenciadoras do desenvolvimento de certos comportamentos infantis, bem como mediadoras dos resultados de intervenções. Porém, foi na década de 90 que surgiram respostas para a preocupação com a manutenção e generalização dos resultados das pesquisas e o destaque dos estudos passou a ser a prevenção e seus tópicos concernentes: fato- res de risco e proteção, redes de apoio, ambiente de convivência da família e sua relação com a criança, a escola e a comunidade.

Rios e Williams (2008) fazem uma revisão de estudos nacionais e internacionais sobre intervenções com famílias afirmando que, apesar de haver diversos estudos de intervenção com pais na realidade brasileira, esses ainda são escassos em comparação a países mais desenvolvidos. Um programa de Treinamento de Habilidades Sociais envolvendo um grupo de quatro casais foi desenvolvido por Bolsoni-Silva, Del Prette, e Del Prette (2000). Por meio do emprego de técnicas de instrução, ensaio comportamental, modelação e uso de tarefas de casa, os participantes apresentaram aumento em habilidades interpessoais tais como a expressão de carinho e atenção contingente, uso de reforçamento positivo, técnicas positivas de resolução de problemas e diminuição no uso de punições após a participação no treinamento. No entanto, problemas de desobediência e agressividade infantil persistiram.

Bolsoni-Silva (2007) descreveu outro procedimento de intervenção em grupo de 14 sessões voltadas para o aumento de repertório de práticas educativas positivas para pais e redução de suas práticas negativas, ampliando assim o repertório de habilidades sociais das crianças. Foi observada a redução de problemas externalizantes apresentados pelas crianças na medida em que as mães indicaram um aumento da expressão de sentimentos, estabelecimento de limites e estabelecimento de consequências para aqueles comportamentos de que não gostavam (Bolsoni-Silva, 2007). Em procedimento semelhante, Bolsoni-Silva e Marturano (2010) avaliaram três grupos, com quatro cuidadoras cada, com procedimento envolvendo medidas de controle pré e pós-intervenção. Em dois meses de intervenção e utilizando como medidas o Inventário de Habilidades Sociais (IHS), um roteiro de avaliação funcional das interações na díade pais e filhos e medidas do Child Behavior Cheklist (CBCL), foi constatada a diminuição nas práticas parentais negativas e redução de problemas de comportamento infantil. Entretanto, não houve mudança significativa do aumento de repertório de práticas parentais positivas em relação à criança. 
Embora fuja do escopo do presente trabalho detalhar todos os estudos ou programa recentes brasileiros de intervenção parental, cabe também mencionar o Programa Parceria, uma proposta de prevenção secundária e terciária de violência planejado para ensinar praticas positivas para mães vítimas de violência intima do parceiro, de forma a prevenir problemas de comportamento em crianças (ver Pereira, D'Affonseca, \& Williams, 2013; Williams, Santini, \& D'Affonseca, 2014). Adicionalmente, Santini, D'Affonseca, Ormeño, e Williams (2012) relatam um estudo de caso utilizando o Projeto Parceira com uma mãe de 43 anos previamente encarcerada. As autoras constataram uma melhora do desempenho da participante em todos os instrumentos aplicados ao longo da intervenção (Potencial de Abuso Infantil - CAP, Inventário de Estilos Parentais IEP e Inventário de Depressão de Beck - BDI), em medidas de pré e pós-teste e follow-up. A partir do relato da participante, foram notadas melhoras quanto a habilidades sociais, resolução de problemas sociais e autoestima após os 20 encontros semanais individuais programados para essa intervenção.

Silvares e Banaco (2000) defendem a relevância de estudos de caso uma vez que eles podem aproximar a pesquisa e a prática. Entretanto, há poucos estudos de caso na literatura nacional que descrevam o percurso do treino parental com pais, com detalhamento de suas técnicas e resultados para que essa modalidade de pesquisa possa realizar a ponte entre teoria e prática. Apesar de não haver consenso de como proceder quanto a relatos de estudos de caso, esse é um dos métodos disponíveis ao clínico e pesquisador para produzir conhecimento na Psicologia (Silvares \& Banaco, 2000). De fato, Peres e Santos (2005) relataram um aumento no emprego regular de estudos de caso nas ciências humanas e sociais. Tal metodologia de estudo, apesar de caráter exploratório e com dados de difícil generalização, pode auxiliar na formulação de hipóteses para pesquisas futuras (Del Prette, Silvares, \& Meyer, 2005).

Em um caso de agressividade infantil em contexto clínico, foi realizada a avaliação de um treino de pais em termos da mudança do comportamento dos pais e seus efeitos sobre o comportamento agressivo de uma criança de 9 anos (Emídio, Ribeiro, \& De-Farias, 2009). Os pais foram orientados a registrar a frequência do comportamento agressivo, bem como, a identificar os antecedentes e consequentes desse comportamento. Após a identificação das contingências que auxiliavam na aquisição e manutenção do comportamento agressivo, os pais foram instruídos a reforçarem os comportamentos adequados, serem consistentes quanto às regras impostas à criança, além de serem ensinados a substituir ameaças por práticas positivas. Dentre os resultados alcançados, observou-se uma diminuição na frequência de comportamentos agressivos verbais e físicos, aumento das habilidades sociais e melhor convívio familiar.

Kaiser (2013) realizou um estudo de caso envolvendo treino de habilidades parentais com mães de duas famílias em descumprimento com os critérios estabelecidos pelo Programa Bolsa Família, com crianças apresentando baixa frequência escolar, além de constatado conflito familiar (como, por exemplo, punição inconsistente e supervisão excessiva por parte da mãe, abuso de álcool pela filha adolescente). Tal treino consistia no ensino do diálogo como técnica de negociação e resolução de conflitos, fortalecimento de vínculos, aplicação de reforço positivo e o estabelecimento e cumprimento de regras. Dados do IEP, respondidos pelos filhos e pelas mães, refletiram em resultados positivos para ambos os casos.

\section{O Programa ACT}

Diferentemente do Projeto Parceria mencionado, o ACT é um programa parental de prevenção universal à violência praticada contra a criança cujo nome completo é ACT - Raising Safe Kids (ACT-RSK) - as iniciais ACT significam Adults and Children Together (Adultos e Crianças em Conjunto). Tal programa foi desenvolvido pela American Psychological Association - APA (Silva, 2011) e tem se mostrado eficaz na prevenção de interações não adaptativas (ou seja, com uso de violência) entre pais e crianças, reduzindo e prevenindo problemas de 
conduta, bem como contribuindo para a diminuição da expressão de comportamentos externalizantes em crianças (Knox, Burkhart, \& Hunter, 2010; Knox et al., 2011). O Programa (doravante denominado ACT) teve o apoio inicial da $\mathrm{Na}$ tional Association for the Education of Young Children (NAEYC) e baseou-se em resultados empíricos de prevenção à violência na infância. Tal programa teve uma versão traduzida para o português: Programa ACT para Educar Crianças em Ambientes Seguros (Silva, 2011). O programa inclui, além de intervenções sócio-cognitivas (instrução didática, modelagem, role-playing), treinamento parental grupal com informações acerca do desenvolvimento da criança, fatores de risco para o desenvolvimento saudável, treino em comunicação e outras habilidades sociais, bem como a resolução de conflitos sem o uso da violência (Knox et al., 2011).

O ACT treina profissionais e famílias a definirem comportamentos infantis apropriados a cada etapa do desenvolvimento, enfatizando a importância de os pais desempenharem um papel positivo para a criança no sentido de monitorar e guiar o comportamento dessa sem o uso da violência ao utilizar práticas pró-sociais. $\mathrm{O}$ programa também enfatiza o envolvimento de pais nas escolas e comunidades para a prevenção da exposição da criança à violência (Knox et al., 2011).

Embora estejam no momento sendo conduzidas estudos sobre o ACT em diversas Universidade no Brasil, ainda não há estudos publicados relativos à eficácia do Programa ACT (Silva \& Williams, 2015). O estudo de caso a ser descrito resultou de uma tentativa inicial frustrada de se aplicar o ACT em grupo (tal como o modelo proposto). Houve dificuldades de adesão ao grupo por motivos variados, relacionados aos desafios de se trabalhar com mães de baixa escolaridade e baixo poder aquisitivo, residentes de bairros vulneráveis. Assim 13 mães inicialmente demonstraram interesse em participar, seis assinaram Termos de Consentimento e apenas duas iniciaram a intervenção. Entretanto, apenas uma mãe, que tinha um perfil contrastante às demais (alta escolaridade e renda), participou de toda a intervenção. Por conseguinte, o presente estudo teve por objetivo relatar a intervenção realizada com tal cuidadora do programa ACT, analisando os dados de tal mãe sobre si mesma e sobre seu filho único e ainda o relato de terceiros (marido da participante e pai da criança).

\section{Método}

\section{Participantes}

Maria (nome fictício), mãe de 36 anos de idade, nível superior completo, profissional na área de Saúde, cursando mestrado e casada foi a participante que completou toda a intervenção. A participante era mãe de filho único de seis anos e possuía renda mensal superior a seis salários mínimos (nível socioeconômico A1 segundo o "Critério Brasil" da Associação Brasileira de Empresas de Pesquisa, 2013). O marido tinha 43 anos, ensino superior completo, também na área de saúde, e pós-graduação.

\section{Local}

O estudo foi conduzido em uma Organização Não-Governamental (ONG) localizada em bairro de grande vulnerabilidade social de uma cidade do interior de São Paulo. A participante do estudo não residia próximo à $\mathrm{ONG}$, sendo informada sobre o programa em ambulatório de saúde da cidade (houve ampla divulgação pela imprensa escrita e por rádio).

\section{Instrumentos}

Questionário de Avaliação do Programa $A C T$, sendo utilizado para medir a satisfação do participante com o programa. São, ao todo, quatro questões: a primeira com subitens a serem preenchidos com a marcação "discordo totalmente", "discordo", "não sei", "de acordo", "totalmente de acordo" quanto a afirmações sobre os facilitadores do programa, a aplicabilidade do mesmo para o dia-a-dia e recomendações acerca da intervenção; a segunda, sobre se o programa teria ajudado o participante a aprender; a terceira, sobre o que o participante teria gostado do ACT; e a quarta questão sobre o que o participante mudaria no programa. 
Inventário de Estilos Parentais - IEP (Gomide, 2006). Faz uma avaliação quanto aos estilos parentais dos cuidadores, classificandoos nos seguintes grupos: estilo parental ótimo quando há expressão de práticas de disciplina positivas e sem manifestação de práticas negativas; estilo parental regular acima da média, no qual a leitura de livros de orientação para pais aprimoraria as práticas parentais manifestas; estilo parental regular abaixo da média, no qual seria interessante que esses pais/cuidadores participassem de grupos de treinamentos parentais; estilo parental de risco, no qual é recomendada a intervenção terapêutica. São avaliadas sete subcategorias com seis itens cada: monitoria positiva, comportamento moral, punição inconsistente, negligência, disciplina relaxada, monitoria negativa e abuso físico, com pontuação mínima total de zero e máxima de 12 por subcategoria.

Adult Self-Report ${ }^{2}$ (ASR) ou Inventário de Autoavaliação para Adultos de 18 a 59 anos (Achenbach \& Rescorla, 2001), permite verificar diversos aspectos de funcionamento adaptativo do adulto, a partir do ponto de vista do próprio participante, podendo fornecer dados relevantes sobre as características da população atendida, bem como, quais os tipos de problema de comportamento que aparecem com mais frequência. No presente trabalho serão analisadas exclusivamente a escala de comportamento agressivo e a escala total de problemas de comportamento. Os problemas são classificados na faixa normal (não-clínica), limítrofe ou clínica e esta escala também identifica psicopatologias baseadas em manuais como o DSM-IV (Achenbach, 2001). Cabe mencionar que os instrumentos ASR e ABCL (ver item abaixo) encontram-se em fase de validação por Silvares (2013), tendo tal autora autorizado o seu uso no presente estudo. Dados de estudos de validação do CBCL (6 a 18 anos), no Brasil, já constam na literatura (Bordin et al., 2013; Rocha et al., 2012).

Versão Brasileira, não publicada do "Adult Self-Report for ages 18-59" (Achenbach \& Rescorla, 2001) traduzida por M. M. Rocha e E. F. M. Silvares (2010). Disponível para compra no seguinte endereço eletrônico: asebabrasil@gmail.com
O Adult Behavior Check-List ${ }^{3}$ (ABCL) ou Inventário de Comportamentos de adultos entre 18 e 59 anos, é respondido por familiares, amigos ou pessoas próximas do adulto e tem por objetivo avaliar os mesmos itens do ASR, para que se possa compará-los, avaliando a diferença entre os respondentes (Achenbach \& Rescorla, 2001).

Child Behavior Checklist ${ }^{4}$ (CBCL), ou "Inventário de Comportamentos de crianças e adolescentes entre 6 e 18 anos" (Achenbach \& Rescorla, 2000) possui 118 itens com subescalas de Ansiedade/depressão; Retraimento/depressão, Queixas somáticas, Problemas de sociabilidade, Problemas de atenção, Problemas com pensamento, Violação de regras e Comportamento agressivo. Tais subescalas são divididas em Escala de internalização (EI) e Escala de externalização (EE). Possui ainda seis escalas em concordância com critérios diagnósticos estabelecidos pelo DSM-IV: Problemas Depressivos, Problemas de Ansiedade, Problemas Somáticos, Problemas de Déficit de Atenção e Hiperatividade, Problemas de Oposição e Desafio e Problemas de Conduta.

Diário de Campo no qual foi feito o registro, a cada sessão, de relatos que indicassem a utilização das estratégias trabalhadas durante a intervenção, bem como sobre a estrutura e aceitação do programa, com a finalidade de acompanhar as mudanças da participante de maneira qualitativa.

\section{Procedimento}

O presente estudo foi aprovado pelo Comitê de Ética em Pesquisa da Universidade (processo 277.394) e os participantes assinaram o Termo

3 Versão brasileira, não publicada do "Adult Behavior Checklist for ages 18-59" (Achenbach \& Rescorla, 2001), traduzida por E. F. M. Silvares e M. M. Rocha (2010). Disponível para compra no seguinte endereço eletrônico: asebabrasil@ gmail.com

4 Versão brasileira, não publicada, do "Child Behavior Checklist for ages 6-18" (Achenbach \& Rescorla, 2001), traduzida por I. A. Bordin, E. F. M. Silvares, M. M. Rocha, M. C. Teixeira e C. S. Paula (2010). Disponível para compra no seguinte endereço eletrônico: asebabrasil@gmail.com 
de Consentimento Livre e Esclarecido. Foi realizada uma parceria entre o Laboratório de Análise e Prevenção da Violência (Laprev) e o Laboratório de Pesquisa em Prevenção de Problemas em Desenvolvimento e Comportamento da Criança (LAPREDES) com a Associação Americana de Psicologia, (APA) no sentido de desenvolver estudos voltados para a validação do ACT para a realidade brasileira. Como resultado, houve um treinamento ministrado pela diretora do Setor de Prevenção da Violência da APA para os pesquisadores dos dois laboratórios, além da tradução do material do ACT para o português.

No Programa ACT são realizadas oito sessões semanais em grupo, com uma média de duas horas de duração cada, abordando as seguintes temáticas: compreendendo o comportamento da criança; a exposição da criança à violência; entendendo e controlando a raiva (dos pais e da criança); resolvendo conflitos de maneira positiva; disciplina positiva e estilos parentais, influência da mídia no comportamento das crianças e a incorporação do Programa no lar e na comunidade. Essas temáticas constituem as quatro estratégias de prevenção do programa em consonância com conhecimentos acerca do desenvolvimento infantil: manejo de raiva, resolução de problemas sociais, disciplina positiva e exposição da criança à mídia/ meios eletrônicos (Silva \& Randall, 2005).

O programa ACT (Silva, 2011) contém diversos manuais para sua aplicação: (a) um Manual para os Pais ou Cuidadores em que há folhas informativas referentes aos assuntos tratados a cada sessão; (b) o Manual do Facilitador, em que se descreve cada encontro do programa, explanando passo-a-passo as atividades para aplicação no grupo; (c) o Manual de Entrevista Motivacional (EM) em que é trabalhada a motivação e nível de prontidão dos participantes para a mudança, sendo tal material apresentado antes da primeira sessão e ao final das sessões 5 e 8 do programa; (d) um Guia de Avaliação.

Considerando as dificuldades previamente mencionadas de se realizar a intervenção em grupo, adaptações do protocolo ACT foram feitas para o formato individual a partir da quarta sessão, quando a segunda participante desistiu (em função da perda de guarda de seu filho). Atividades grupais tais como role-playing e confecção de cartazes foram realizadas com o auxílio das facilitadoras que desempenharam papel de participantes. Pelo fato de a intervenção ser individual, sem portanto relatos de outros participantes, situações de interação da própria participante com seu filho tornaram-se exemplos para a aplicação de estratégias e conceitos a serem aprendidos nas folhas informativas do Manual dos Pais.

O procedimento consistiu em quatro fases: (a) pré-intervenção (duas sessões de aproximadamente duas horas de duração), na qual foi aplicado o IEP, ASR, CBCL, ABCL e CBCL tanto na participante como em seu marido; (b) intervenção, que consistiu nas oito sessões do programa ACT realizadas no período noturno uma vez por semana, com duas horas de duração cada e com um intervalo de 15 minutos; (c) pós-intervenção (duas sessões de duas horas de duração), na qual foram reaplicados todos os instrumentos em Maria e seu marido, sendo também verificada a opinião da mesma sobre o ACT a partir do Questionário de Avaliação do Programa; (d) follow-up (duas sessões de duas horas de duração) três meses após a intervenção em que se verificou a estabilidade dos resultados obtidos pela reaplicação dos instrumentos em Maria e seu marido. Durante a primeira fase, os instrumentos foram preenchidos em grupo (portanto com outras mães que assinaram o Termo de Consentimento) e, nas fases seguintes, os instrumentos foram preenchidos individualmente.

Aplicaram o Programa ACT duas psicólogas e mestrandas em Programa de Pós-Graduação em Psicologia, sendo uma delas a primeira autora do estudo. Durante o curso, era oferecido um lanche e monitoria para a criança no período do curso. Além disso, pequenos incentivos foram oferecidos durante a intervenção, como doces, livros e brinquedos infantis, etc.

\section{Análise de Dados}

Para o IEP foram considerados os escores totais obtidos pela mãe a cada preenchimento, sendo considerada a sua classificação em cada fase do estudo. Para os instrumentos ASEBA 
(Achenbach System of Empirically Based Assessment, instrumentos de Achenbach) foram verificadas as escalas de comportamento agressivo da mãe e da criança e a escala de problemas totais de comportamento relativos a ambos. Para análise de dados foram considerados dados que indicaram mudança de comportamento do pré para o pós-teste e follow-up ou que tiveram discordância entre os informantes em algum desses períodos considerados. Os dados dos instrumentos ASEBA foram computados pelo Assessment Data Manager (ADM), software especialmente desenvolvido por Achenbach para esse fim (2006).

\section{Resultados}

Na fase de pré-teste a participante verbalizou que estava tendo problemas no seu relacionamento conjugal e sinalizou a possibilidade de se divorciar.

\section{IEP}

Analisando os escores obtidos pela avaliação de Maria no IEP, verifica-se que, ao realizar o pré-teste, a cuidadora foi classificada como apresentando "estilo parental regular acima da média" (escore total $=9$, percentil 75). Ao ser avaliada no pós-teste, Maria foi classificada como "estilo parental ótimo" (escore total $=12$, percentil 86), no qual há a presença marcante de práticas parentais positivas e ausência das negativas, permanecendo nessa classificação na medida de follow-up (escore total $=14$, percentil entre 90-96) três meses após a sua participação no grupo.

\section{ASEBA}

A Tabela 1 a seguir apresenta os resultados da mãe e da criança nos instrumentos do sistema ASEBA. Houve concordância entre o relato dos pais quanto à avaliação da criança no CBCL. A criança apresentou escores em nível não-clínico no pré-teste quanto ao comportamento agressivo, mantendo tal nível ao longo de todo o estudo (pós-teste e follow-up). Quanto ao escore total de problemas, esses foram mantidos em nível não-clínico no pós-teste, havendo uma queda para a faixa limítrofe na fase de follow-up.

Houve, de acordo com a Tabela 1, no geral, discrepância entre os relatos da participante em sua autoavaliação no ASR e a avaliação feita por seu marido no ABCL quanto aos aspectos de seu funcionamento adaptativo e problemas comportamentais avaliados por esses instrumentos. No autorrelato da participante, houve melhoras rela-

Tabela 1

Desempenho da Mãe e Criança nos Instrumentos ASEBA Considerando o Relato de Dois Informantes

\begin{tabular}{|c|c|c|c|c|c|c|c|c|c|c|c|}
\hline \multirow{6}{*}{ 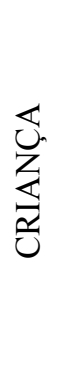 } & \multirow{2}{*}{ Subescalas } & & \multicolumn{3}{|c|}{ Pré-teste } & \multicolumn{3}{|c|}{ Pós-teste } & \multicolumn{3}{|c|}{ Follow-up } \\
\hline & & & Escore & Percentil & & Escore & Percentil & & Escore & Percentil & \\
\hline & Comportamento & Mãe & 53 & 62 & $\mathrm{~N}$ & 50 & $<=50$ & $\mathrm{~N}$ & 57 & 76 & $\mathrm{~N}$ \\
\hline & Agressivo & Pai & 54 & 65 & $\mathrm{~N}$ & 53 & 62 & $\mathrm{~N}$ & 61 & 87 & $\mathrm{~N}$ \\
\hline & \multirow{2}{*}{ Total de Problemas } & Mãe & 55 & 69 & $\mathrm{~N}$ & 47 & 38 & $\mathrm{~N}$ & 61 & 87 & B \\
\hline & & Pai & 51 & 64 & $\mathrm{~N}$ & 51 & 64 & $\mathrm{~N}$ & 60 & 84 & B \\
\hline \multirow{6}{*}{ 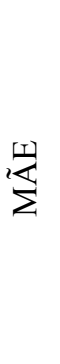 } & \multirow{2}{*}{ Subescalas } & & \multicolumn{3}{|c|}{ Pré-teste } & \multicolumn{2}{|r|}{ Pós-teste } & & \multicolumn{3}{|c|}{ Follow-up } \\
\hline & & & Escore & Percentil & & Escore & Percentil & & Escore & Percentil & \\
\hline & \multirow{2}{*}{$\begin{array}{c}\text { Comportamento } \\
\text { Agressivo }\end{array}$} & Maria & 56 & 73 & $\mathrm{~N}$ & 60 & 84 & $\mathrm{~N}$ & 65 & 97 & B \\
\hline & & Marido & 75 & $>97$ & $\mathrm{C}$ & 84 & $>97$ & $\mathrm{C}$ & 69 & 97 & B \\
\hline & \multirow{2}{*}{ Total de Problemas } & Maria & 65 & 93 & $\mathrm{C}$ & 59 & 81 & $\mathrm{~N}$ & 65 & 93 & $\mathrm{C}$ \\
\hline & & Marido & 86 & $>98$ & $\mathrm{C}$ & 89 & $>98$ & $\mathrm{C}$ & 67 & 96 & $\mathrm{C}$ \\
\hline
\end{tabular}

Note. $\mathrm{C}=$ Clínica, $\mathrm{L}=$ Limítrofe, $\mathrm{N}=$ Não clínico. 
tivas ao escore total de problemas, mudando sua classificação da faixa clínica (pré-teste) para a normal (pós-teste). Em contraste, de acordo com o marido, a participante permaneceu na faixa clínica em todas as fases de avaliação, não indicando melhoras quanto a esses aspectos. Quanto às medidas de follow-up relativas ao comportamento agressivo, Maria se autoavaliou na faixa limítrofe, em concordância com o marido que também a situou nessa faixa de classificação.

Segundo sua auto-avaliação, Maria mantinha um nível de funcionamento adaptativo não-clínico, indicando ausência de agressividade (não-clínica), no pré e pós-teste, sem manutenção no follow-up, quando houve uma queda em seu desempenho (limítrofe). A avaliação do informante (marido) a manteve nos escores a nível clínico no pré e pós-teste, havendo uma melhora três meses após a intervenção (follow-up). Ainda de acordo com o mesmo informante, os escores totais de problemas externalizantes e internalizantes de Maria mantiveram-se a nível clínico em todas as fases de avaliação enquanto a participante relatou melhoras após a intervenção, ainda que tal mudança não tenha se estabilizado na fase de follow-up.

\section{Questionário de Avaliação do Programa ACT}

Maria já apresentava antes de iniciar o curso, segundo o Guia de Avaliação ACT, conhecimento acerca das temáticas trabalhadas. Em todas as escalas, na fase de pré-intervenção, pós-intervenção e follow-up, ela apresentou escores acima da média, referentes aos temas trabalhados no Programa, sem variações importantes entre as fases apresentadas. No Questionário de Avaliação do Programa, a mãe relatou que os facilitadores tinham domínio sobre o assunto, eram amáveis e eficientes. Ressaltou ainda que gostou do programa, pois esse lhe forneceu boas opções para lidar com seu filho, afirmando que iria utilizar as técnicas que aprendeu. Apesar de achar o programa longo, recomendaria o programa a outras pessoas e relatou que os encontros ajudaram-na a refletir sobre os temas e a relembrá-los a cada encontro.

\section{Diário de Campo}

Conforme mencionado, a participante relatou durante o preenchimento dos instrumentos ASEBA, no pré-teste, dificuldades quanto ao relacionamento conjugal. Citou como motivo de interesse no curso o fato de outras pessoas acharem que ela não colocava limites na criança, além do desejo de "gritar menos" com seu filho. Durante a intervenção, na segunda sessão, a mãe teve dificuldades em descrever os tipos de violência que possam existir (violência sexual, física, psicológica e negligência), tendo certa dificuldade para defini-las, sobretudo quanto à negligência. Nessa mesma sessão, a mãe relatou ter ficado nervosa durante uma dinâmica, em que se rasga um pedaço de um boneco de papel a cada fator de risco que uma criança poderia experienciar e informou compreender que esses diversos tipos de violência acabam deixando marcas na criança.

A mãe relatou que os modelos de resolução de conflitos propostos pelo Programa a auxiliaram a se acalmar antes de tomar decisões quanto à maneira de disciplinar seu filho. Maria relatou ter impresso cópias de alguns dos materiais recebido para espalhar em casa de modo a auxiliá-la a se lembrar de não agir de maneira impulsiva. Maria ressaltou ainda, durante a sessão sobre meios eletrônicos, que "nunca tinha parado para pensar" nos exemplos de "heróis" que deixamos nossos filhos expostos nos jogos de videogame e na televisão e que poderíamos buscar em nossa comunidade, verdadeiros exemplos de heróis. "Toda situação é uma oportunidade pra ensinar, é isso? Achei bem legal essa história do herói mesmo...", disse.

Maria reconheceu durante as discussões sobre estilos parentais que muito do que ela fazia para educar seu filho era, de alguma forma, fruto da educação que tivera. No entanto, disse que não identificava essa relação anteriormente $\mathrm{e}$ que o Programa a auxiliara a compreender certos comportamentos que apresentava. Na sexta sessão, Maria relatou que seu filho estava conversando mais com ela e relatando fatos que estavam acontecendo na escola. (Na primeira sessão, Maria havia identificado como um problema o 
fato de o filho não conversar muito com ela). A participante relatou, ainda, que sua relação com o filho estava mais próxima e disse estar feliz com estes resultados.

A mãe relatou, no pós-teste, que optou por iniciar o processo de separação. Disse também que havia conseguido manter a boa relação com o filho no período de pós-teste, consultando o material fornecido durante o curso. $\mathrm{Na}$ fase de follow-up, a mãe relatou que concluíra o processo de separação, dizendo-se feliz com sua decisão. Relatou, ainda, que, com a melhora no diálogo com o filho, conseguiu introduzir o assunto da separação com a criança, na tentativa de minimizar os efeitos de tal processo em seu desenvolvimento.

\section{Discussão}

Maria participou de todas as sessões do programa, sem faltas. Sua total adesão ao programa foi de certo modo surpreendente, pois para participar do ACT, realizava, semanalmente, um percurso longo indo de sua residência a um bairro distante na periferia da cidade. Tal adesão pode ter sido favorecida por: (a) sua alta escolaridade e renda (pais com tais características têm melhor adesão a programas de treinamento; Webster-Stratton, 1998); (b) sua motivação em lidar com uma crise que a família enfrentava em decorrência da separação e (c) os benefícios que possivelmente obteve ao participar do Programa ACT.

Quanto a esses benefícios, os dados do IEP sugerem que houve um aumento de práticas positivas desempenhadas pela mãe, tema trabalhado em detalhes com a participante durante o módulo de disciplina positiva, fornecido pelo protocolo do programa. A estabilidade de dados no follow-up, obtidos com um instrumento validado com medidas anteriores e posteriores à intervenção, sugere que, a intervenção pode ter realmente levado à mudança, ainda que o estudo consista em um estudo de caso (Kazdin, 2003). Tais resultados são fortalecidos, de certo modo, com os dados qualitativos demonstrando que a participante avaliou o Programa ACT de modo favorável e não faltou a qualquer uma das sessões do mesmo.

Os mesmos benefícios nas práticas positivas e melhora no Estilo Parental com escores indo de Acima da Média para Ótimo não foram observados nos dados coletados pelo marido de Maria. Tal discrepância pode ter sido maximizada pelo fato de o casal estar mais propenso a avaliar o outro negativamente, pois estavam em processo de separação. Uma coleta de dados com um Protocolo de Observação poderia confirmar ou não se tal hipótese estaria correta, sendo recomendável em futuros estudos, bem como uso de delineamentos de pesquisa mais complexos.

Quanto ao desempenho da criança, essa já apresentava comportamento adaptativo no CBCL, situado na faixa não clínica nas escalas avaliadas pelo instrumento desde o pré-testes, segundo ambos informantes, dessa forma, o efeito teto indicava a não possibilidade de melhora. Seria interessante, assim a coleta de dados com informantes adicionais, como professores, para completar tal constatação e fornecer uma avaliação externa.

$\mathrm{O}$ fato de Maria estar em processo de separação era motivo de estresse e em si já seria um fator a justificar a necessidade de participar de um Programa parental. De fato, a literatura constata que o estresse parental é uma das variáveis influentes no desempenho familiar em programas de intervenção (Farrelly \& Mclennon, 2010; Mytton, Ingram, Manns, \& Thomas, 2014). Williams e Aiello (2004) citam o livro de McKenry e Price (1994) no qual os autores reveem as principais formas de estresse a ser enfrentadas por famílias, lembrando-nos de que o divórcio é pontuado como um dos piores eventos estressores. A literatura aponta, também, que é menos provável que as habilidades parentais adequadas se mantenham em um ambiente com crises maritais e o contexto de separação, aumentaria a probabilidade de os pais e as crianças exibirem problemas de comportamento (Amato, 2000; Dadds, Schwartz, \& Sanders, 1987; Kelly \& Emery, 2003; Raposo et al., 2011). Nesse sentido, a qualidade da relação conjugal dos pais participantes em programas parentais pode 
influenciar o desempenho dos mesmos (Moran, Ghate, \& Van der Merwe, 2004), a exemplo dos resultados discrepantes obtidos nos instrumentos ASEBA.

O presente estudo de caso é útil para ilustrar que, tal como se pretende em um programa universal de prevenção de violência, o Programa ACT foi benéfico à Maria (segundo seu relato e dados coletados), a despeito do fato de ela ser mãe com alta escolaridade e renda. Assim, o ACT parece ter servido para Maria aprimorar o seu estilo parental, dando-lhe conhecimentos e informações novas sobre práticas parentais positivas. Adicionalmente, embora não seja o foco do Programa, ele pareceu ter auxiliado Maria a lidar com as questões envolvendo seu filho durante a crise de separação, tranquilizando-a e auxiliando-a a resolver problemas de modo não violento (essa, sim, uma das metas do Programa).

Cabe esclarecer, entretanto, que o fato de Maria ter alta escolaridade tenha possivelmente facilitado a adesão não signifique que o Programa ACT tenha sucesso apenas com cuidadores com tais características. Ao reverem a implantação do Programa ACT, Silva e Williams (2005) informam que o mesmo é utilizado com sucesso em populações distintas de diversos países, conclusão semelhante a de Altafim e Linhares, 2016.

Estudos de caso são importantes para o desenvolvimento de hipóteses sobre problemas clínicos e para conhecer mais a fundo tratamentos inovadores (Kazdin, 2003), apesar das dificuldades para a generalização dos dados encontrados. Além disso, tais estudos podem facilitar o trabalho para futuras investigações com delineamentos distintos (Del Prette et al., 2005). Sugerem-se, dessa maneira, trabalhos futuros com o Programa ACT no Brasil com intervenções em delineamentos de grupo e ensaios clínicos randomizados mantendo a utilização de múltiplos informantes, com a finalidade de substanciar os dados obtidos. Adicionalmente, sugere-se a utilização de instrumentos adicionais para coleta de dados, tal como no presente estudo, uma vez que os instrumentos de avaliação do ACT resultam apenas em dados qualitativos.

\section{Referências}

Achenbach, T. M. (2001). Manual for the Data Manager Program (ADM): CBCL, YRS, TRE, $Y A R S, \quad Y A B C L, \quad C B C L / 2-3, \quad C B C L / 1 / 2-5 \quad \&$ $C-T R F$. Burlington, VT: Achenbach System of Empirically Based Assessment.

Achenbach, T. M., \& Rescorla, L. A. (2000). Manual for the ASEBA Preschool Forms \& Profiles. Burlington, VT: University of Vermont, Research Center for Children, Youth, \& Families.

Achenbach, T. M., \& Rescorla, L. A. (2001). Manual for the ASEBA Adult Forms \& Profiles. Burlington, VT: University of Vermont, Research Center for Children, Youth, \& Families.

Achenbach System of Empirically Based Assessment. (2006). Manual for the Assessment Data Manager Program (ADM). Burlington, VT: University of Vermont.

Altafim, E.R.P. \& Linhares, M.B.M. (2016). Universal violence and child maltreament prevention programs for parents: A systematic review. Psychosocial Intervention, 25 (1), 27-38.

Amato, P. R. (2000). The consequences of divorce for adults and children. Journal of Marriage and the Family, 62, 1269-1280. doi:10.1111/j.17413737.2000.01269.x

Associação Brasileira de Empresas de Pesquisa. (2013). Critério de Classificação Econômica Brasil. Recuperado em http://www.abep.org/ criterio-brasil

Bolsoni-Silva, A. T. (2007). Intervenção em grupo para pais: Descrição de procedimento. Temas em Psicologia, 15(2), 217-235.

Bolsoni-Silva, A. T., Del Prette, A., \& Del Prette, Z. A. P. (2000). Relacionamento pais-filhos. Um Programa de desenvolvimento interpessoal em grupo. Psicologia Escolar e Educacional, 3(3), 203-215.

Bolsoni-Silva, A. T., \& Marturano, E. M. (2002). Práticas educativas e problemas de comportamento: Uma análise à luz das habilidades sociais. Estudos de Psicologia (Natal), 7(2), 227-235. doi:10.1590/S1413-294X2002000200004

Bolsoni-Silva, A. T., \& Marturano, E. M. (2010). Evaluation of group intervention for mothers/ caretakers of kindergarten children with externalizing behavioral problems. Interamerican Journal of Psychology, 44(3), 415-421. 
Bordin, I. A., Rocha, M. M., Paula, C. S., Teixeira, M. C. T. V., Achenbach, T. M., Rescorla, L. A., \& Silvares, E. F. M. (2013). Child Behavior Checklist (CBCL), Youth Self-Report (YSR) e Teacher's Report Form (TRF): Uma visão geral sobre o desenvolvimento das versões originais e brasileiras. Cadernos de Saúde Pública, 29(1), 13-28. doi:10.1590/S0102311X2013000100004

Carvalho, M. C. N., \& Gomide, P. I. C. (2005). Práticas educativas parentais em famílias de adolescentes em conflito com a lei. Estudos de Psicologia (Campinas), 22(3), 263-275. doi:10.1590/ S0103-166X2005000300005.

Dadds, M. R., Schwartz, S., \& Sanders, M. R. (1987). Marital discord and treatment outcome in behavioral treatment of child conduct disorders. Journal of Consulting and Clinical Psychology, 55, 396-403. doi:10.1037/0022-006X.55.3.396

Del Prette, G., Silvares, E. F. M., \& Meyer, S. B. (2005). Validade interna em 20 estudos de caso comportamentais brasileiros sobre terapia infantil. Revista Brasileira de Terapia Comportamental e Cognitiva, 7(1), 93-105.

Dubowitz, H. (2012). The safe environment for Every Kid (SEEK) model: Child healthcare professionals helping prevent child maltreatment. In H. Dubowitz (Ed.), World perspectives on child abuse (10 ${ }^{\text {th }}$ ed., pp. 89-92). Aurora, CO: International Society for Prevention of Child Abuse and Neglect.

Emídio, L. A. S., Ribeiro, M. R., \& De-Farias, A. K. C. R. (2009). Terapia infantil e treino de pais em um caso de agressividade. Revista Brasileira de Terapia Comportamental e Cognitiva, 11(2), 366-385.

Farrelly, A. C., \& Mclennan, J. D. (2010). Participation in a Parent Education Programme in the Dominican Republic: Utilization and barriers. Journal of Tropical Pediatrics, 56(3), 149-158. doi:10.1093/tropej/fmp071

Gomide, P. I. C. (2003). Estilos parentais e comportamento anti-social. In Z. Del Prette \& A. Del Prette (Eds.), Habilidades sociais, desenvolvimento e aprendizagem: Questões conceituais, avaliação e intervenção (pp. 21-60). Campinas, SP: Alínea.

Gomide, P. I. C. (2006). Inventário de Estilos Parentais (IEP): Modelo teórico, manual de aplicação, apuração e interpretação. Rio de Janeiro, RJ: Vozes.
Kaiser, F. A. (2013). Treino de habilidades parentais: Estudo de caso com famílias em descumprimento de condicionalidades do Programa Bolsa Família (Dissertação de mestrado, Universidade Estadual de Londrina, PR, Brasil).

Kazdin, A. E. (2003). Drawing valid inferences from case studies. In A. Kazdin (Ed.), Methodological issues and strategies in clinical research ( $p \mathrm{p}$. 655-669). Washington, DC: American Psychological Association.

Kelly, J. B., \& Emery, R. E. (2003). Children's adjustment following divorce: Risk and resilience perspectives. Family Relations, 52, 352-362. doi:10.1111/j.1741-3729.2003.00352.x

Knox, M. S., Burkhart, K., \& Hunter, K. E. (2010). ACT Against Violence Parents Raising Safe Kids Program: Effects on maltreatment-related parenting behaviors and beliefs. Journal of Family Issues, 32, 55-74. doi:10.1177/01925 $13 \times 10370112$

Knox, M., Burkhart, K., \& Howe, T. (2011). Effects of the ACT Raising Safe Kids Parenting Program on children's externalizing problems. Interdisciplinary Journal of Applied Family Studies - Family Relations, 60, 491-503. doi:10.1111/j.1741-3729.2011.00662.x

Mejia, A., Calam, R., \& Sanders, M. R. (2012). A review of parenting programs in developing countries: Opportunities and challenges for preventing emotional and behavioral difficulties in children. Clinical Child and Family Psychology Review, 15, 163-175. doi:10.1007/s10567-0120116-9

Moran, O., Ghate, D., \& Van der Merwe, A. (2004). What works in parenting support: A review of the international evidence (Research Report No. 574). London: Department for Education and Skills.

Mytton, J., Ingram, J., Manns, S., \& Thomas, J. (2014). Facilitators and barriers to engagement in parenting programs: A qualitative systematic review. Health Education \& Behavior, 4(2), 127-137. doi:10.1177/1090198113485755

Pereira, P. C., D’Affonseca, S. M., \& Williams, L. C. A. (2013). A feasibility pilot intervention program to teach parenting skills to mothers of polyvictimized children. Journal of Family Violence, 28, 5-15. doi:10.1007/s10896-012-9490-9

Peres, R. S., \& Santos, M. A. (2005). Considerações gerais e orientações práticas acerca do emprego 
de estudos de caso na pesquisa científica em psicologia. Interações, 10(20), 109-126.

Prada, C. G., \& Williams, L. C. A. (2010). Intervenção em práticas educativas para pais e cuidadores em abrigo: Uma revisão. In L. C. A. Williams, J. M. D. Maia, \& K. S. A. Rios (Eds.), Aspectos psicológicos da violência: Pesquisa e intervenção cognitivo-comportamental (pp. 271 -290). Santo André, SP: ESETec.

Raposo, H. S., Figueiredo, B. F. C., Lamela, D. J. P. V., Nunes-Costa, R. A., Castro, M. C., \& Prego, J. (2011). Ajustamento da criança à separação ou divórcio dos pais. Revista de Psiquiatria Clínica, 38(1), 29-33. doi:10.1590/S010160832011000100007

Rios, K. S. A., \& Williams, L. C. A. (2008). Intervenção com famílias como estratégia de prevenção de problemas de comportamento em crianças: Uma revisão. Psicologia em Estudo, 13(4), 799806. doi:10.1590/S1413-73722008000400018

Rocha, M. M., Rescorla, L. A., Emerich, D. R., Silvares, E. F. M., Borsa, J. C., Araújo, L. G. S., ...Assis, S. G. (2012). Behavioural/emotional problems in Brazilian children: Findings from parent's reports on the Child Behavior Checklist. Epidemiology and Psychiatric Sciences, 22(4), 1-10. doi:10.1017/S2045796012000637

Santini, P. M., D’Affonseca, S. M., Ormeño, G. R., \& Williams, L. C. A. (2012). Violência doméstica e encarceramento: Um estudo de caso. Multiciência (ASSER), 11, 212-222.

Silva, J. (2011). ACT Raising Safe Kids Program. Washington, DC: American Psychological Association.

Silva, J. A., \& Williams, L. C. A. (2015). O programa ACT para educar crianças em ambientes seguros: Da elaboração à avaliação. In S. G. Murta, C. Leandro-França, K. B. Santos, \& L. Polejack (Eds.), Prevenção e promoção em Saúde Mental: Fundamentos, planejamento e estratégias de intervenção (pp. 489-300). Novo Hamburgo, RS: Sinopsys.

Silva, J. M., \& Randall, A. (2005). Giving Psychology away: Educating adults to ACT against early childhood violence. Journal of Early Childhood \& Infant Psychology, 1, 37-43.
Silvares, E. F. M. (2013). Estudo de validação multicultural do "Inventário de Autoavaliação para Adultos" (ASR) e do "Inventário de avaliação de Adultos de 18 a 59 anos" (ABCL): Dados brasileiros (Relatório apresentado à Fundação de Amparo à Pesquisa do Estado de São Paulo). Dados brutos não publicados.

Silvares, E. F. M., \& Banaco, R. A. (2000). O estudo de caso clínico comportamental. In E. F. M. Silvares (Ed.), Estudos de caso em Psicologia Clínica Comportamental Infantil (7. ed., Vol. 1, pp. 31-48). Campinas, SP: Papirus.

Webster-Stratton, C. (1998). Parent training with low-income families. In J. Lutzker (Ed.). Handbook of child abuse research and treatment (pp.183-210). New York: Plenum.

Williams, L. C. A., \& Aiello, A. L. R. (2004). Emponderamento de famílias: $\mathrm{O}$ que vem a ser e como medir. In E. G. Mendes, M. A. Almeida, \& L. C. A. Williams (Eds.), Temas em Educação Especial: Avanços recentes (pp. 197-202). São Carlos, SP: EDUFSCar.

Williams, L. C. A., Santini, P. M., \& D’Affonseca, S. M. (2014). The Parceria Project: A Brazilian parenting program to mothers with a history of intimate partner violence. International Journal of Applied Psychology, 4(3), 101-107. doi:10.5923/j.ijap.20140403.04

Wolfe, D. A., \& Jaffe, P. G. (1999). Emerging strategies in the prevention of domestic violence. In The future of children: Domestic violence and children (pp. 133-144). Los Altos, CA: The David and Lucile Packard Foundation.

World Health Organization. (2009). Preventing violence through the development of safe, stable and nurturing relationships between children and their parents and caregivers (Series of Briefings on Violence Prevention: The Evidence). Geneva, Switzerland: Author. 